\title{
Virtual Art Experience on Cultural Heritage Information with Construction of Evaluation Criteria
}

\author{
Chao Wen ${ }^{1, a,{ }^{*}, X i ~ L u o}{ }^{2, b}$ \\ 1,2 Art School, Northwest University, Xi'an, China 710069 \\ asuperheat@163.com, bluoboo77@126.com \\ ${ }^{*}$ Corresponding author
} Keywords: Cultural Heritage Information, Virtual Art, Immersive Experience, Emperor Qin's
Mausoleum

\begin{abstract}
The cultural heritage intuitively reflects the progress of human and social development. As humans having entered the 21st century, an experience era of information pluralism, new information and interaction technologies such as virtual reality (VR) etc. provide effective methods on the digital inheritance of cultural heritage information (CHI). Moreover, the application of virtual art in the digital survival of CHI changes the linear narrative mode of traditional exhibition and propagation and strengthens the interactive narrative characteristic of the theme and story of CHI. The evaluation of virtual art experience has become one of the important measures for promoting the digital development of cultural heritage. Aiming at CHI's virtual art experience, in this paper, firstly a restoration and display VR system of Emperor Qin's mausoleum has been built. Then based on the features of the built VR system, some virtual art characteristics and user experience requirements of the system are analyzed, and an architecture of objective evaluation criteria of virtual art experience is established. Finally, an evaluation test of the virtual art experience on the virtual environment of the First Emperor's mausoleum is adopted according to the criteria, which provides many possibilities for digital protection and reconstruction of CHI in the Qin Dynasty.
\end{abstract}

\section{Introduction}

Due to the physical nature of the cultural heritage, the physical space for exhibition is strictly limited. Traditional display methods pay more attention to the research and protection of cultural relics. One-way information transmission does not take into account the reading ability of audiences of all ages. Therefore, the educational and sharing function of cultural heritage resources does not been effectively developed yet. At present, the design of virtual art has promoted the protection and propagation of digital cultural heritage. With the development of virtual reality (VR) technology, the application and research of virtual arts in cultural heritage at home and abroad can be divided into the following three main aspects: firstly, digital recording and preservation, that is, orderly organizing cultural heritage information (CHI) through data acquisition analysis. For example, "Digital Dunhuang" [1], which was designed by Zhejiang University and Dunhuang Research Institute, allows viewers to enjoy and learn HD digital mural images of Dunhuang; Secondly, digital restoration and recovery, that is, using computer graphics, arts and other knowledge to reconstruct the damaged or lost cultural heritage on the basis of historical facts. For example, "Digital Yuanmingyuan"[2], which was developed by Beijing Institute of Technology, restores the lost Yuanmingyuan on reality ruins with three-dimensional visualization technologies; finally, digital display and interaction, that is, adopting virtual reality including augmented reality methods to interact in a virtual environment. For example, "Virtual Forbidden City" [3], which was released by the Palace Museum, enables viewers to virtually experience and interact with the culture heritage through immersive VR devices.

The main goal of CHI's digital display and experience is to build a virtual environment with sufficient information resources so that the audience (users) can make immersive interactions with 
the virtual environment and obtain the tangible historical information and intangible cultural information of cultural heritage. Based on the above understanding, we conduct our researches on the digital display design and experience evaluation of $\mathrm{CHI}$ under the virtual environment. In this paper, based on the Emperor Qin's mausoleum VR environment we built, we propose an objective evaluation architecture of user experience corresponding to the built virtual environment, and adopt user research methods to assess the interaction and art experience of the virtual environment.

\section{CHI virtual art design of Emperor Qin's mausoleum}

At present, the virtual art form in the field of art and culture is a virtual scenario constructed by the creators and designers, which transmits the naturally visual perception and artistic experience to the viewer. The classical frescoes in the ancient city of Pompeii dating back more than 2,000 years obliterate the barriers between viewers and murals by blurring the boundary between real space and image space and using illusion elements to create full immersion experiences for viewers. Classical frescoes are seen as the primitive form of virtual art. Through composition skills, they create a space integrating with the viewers and change the independent and isolated state of traditional art display. In the 1980s, with the innovation of virtual reality including augmented reality and other intelligent interaction methods, the application category of virtual art has continuously expanded which brings a brand-new sensory experience to viewers and allows the expansion and extension of display and propagation forms on cultural resources.

\subsection{Spatial composition and cultural connotation of Emperor Qin's Mausoleum}

The Emperor Qin's mausoleum, mausoleum of the First Emperor of China (referred to as Qinling), is located in the northern foothills of Lishan Mountain, 5 kilometers east of Lintong District, Xi'an, Shaanxi Province of China. The Qinling was built in the first year of the Qin Dynasty (2,000 years ago). It is the first large-scaled and well-designed imperial mausoleum in Chinese history. Qinling is divided into two parts: the surface building and the underground palace. The total area of Qinling is about 56.25 square kilometers. The world-famous Terracotta Warriors and Horses is one of the burial pits of Qinling. Buildings once surrounded the tomb of Qinling, but after several times' human destruction and storm erosion in the past 2,000 years, the surface remnants of the mausoleum just remain some wall ruins around the sealed earthwork of the existing tomb of Qinling. According to archeological exploration, the Emperor Qin's mausoleum is designed strictly by the palace system of Qin dynasty and its sealed tomb surrounded by the symmetrical layout gongs is the center of Qinling. The surface building of Qinling has a horizontal partition wall that divides surface building into two parts, "the front" and "the back". The huge ladder-like "fighting bucket type sealed earthwork" facing south symbolizes the Front Hall of the imperial court. The Rest Hall relics are in the north direction of the sealed tomb. The Harem Hall and some annexed buildings relics are in the west of the tomb. In the east of the sealed tomb, some relics of accompanied tombs had been found and presumed to be the tombs of concubines who had no children in the harem. Qinling reflects the economic foundation, class relations, social customs and aesthetic concepts of the Qin Dynasty. Thus, it has significant historical, cultural, and artistic values toward the study of human and society of Qin.

\subsection{Qinling virtual restoration and display system}

The digital protection and reconstruction of Qinling consists of two stages of work, surface environment restoration and underground palace construction. Now our Qinling virtual restoration and display system is the research result of the first stage of the work. Based on archaeological data and academic researches, the surface building of Qinling has been completely restored in the virtual restoration and display system. For building the system, Qinling's surface building and its interior spatial structure are created first in the 3D modeling software, as shown in Figure 1. Then, based on the methods of interactive system design, the Qinling VR environment is implemented, which artistically reproduces the historical scene of Emperor Qin's Mausoleum and enables viewers to 
obtain the immersive experience through wearing a VR head-mounted display device and operating the handle. The established system lets viewers interact with the important historical and cultural relics personally and achieve awareness and knowledge in virtual scenarios, as shown in Figure 2.

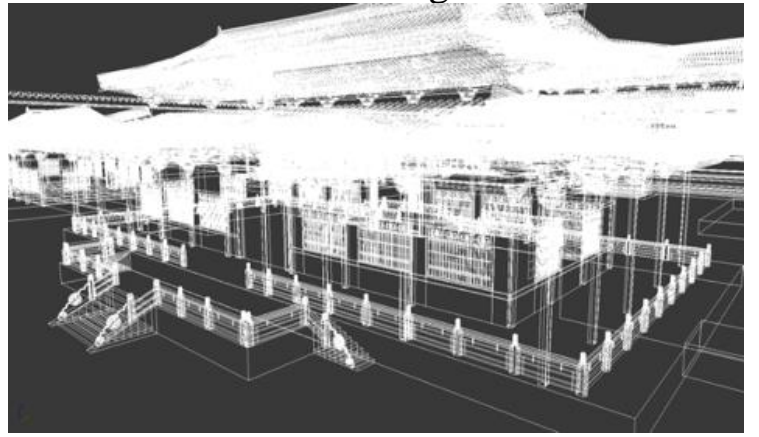

Fig. 1. Block diagram of the main hall of the building group

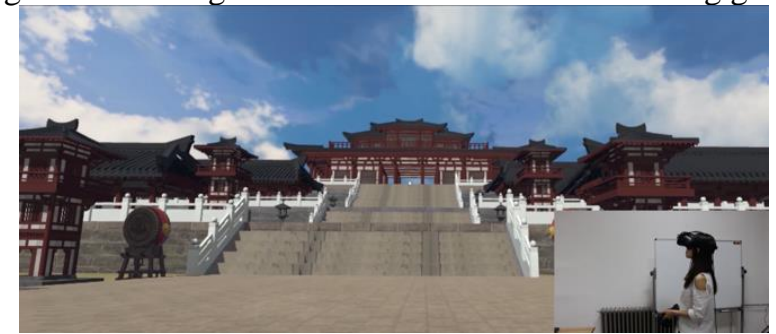

Fig. 2. The virtual restoration and display system of Qinling (users experience in the head-mounted device)

The virtual environment we build has its distinctive space and visual features. Firstly, based on field surveys and academic research data of the mausoleum's architectural site, virtual scene design and layout are arranged according to the objective location of Qinling. Secondly, based on historical records of the Qin Dynasty, the pattern of buildings group is established on the layout of the ancient Xian Yang City (capital city) of Qin, meanwhile the materials of the buildings are mainly woods with dark red and dark gray colors. In addition, the tone and entourage of landscape elements of the virtual environment are combined reasonably to create a visual aesthetic feeling, etc. Thus, users in the environment of virtual Qinling not only have a better immersive experience but also contact with the historical and cultural information through the virtual art forms.

\section{Virtual art experience analysis and evaluation criteria construction}

The user (audience) is the recipient and practitioner of the virtual art experience. "Experience" is the good feeling that comes from a person's consciousness when he reaches a certain level of emotion, physical strength, intelligence, or even spirit [4]. User Experience (UE or UX), including the degree of preference, psychological reflection, emotional factors, cognitive impressions and other aspects, is the subjective experience of the user in the process of experience. Man, the creator and designer of products (systems), is the ultimate user. The core idea of user experience is to focus on the user and clarify the user's expectation and attitude towards the product [5]. This could be extended to the virtual art display of cultural heritage, that is, a good virtual art experience is an important way for $\mathrm{CHI}$ to be interpreted and spread. Therefore, the consideration of UE's essential factors and construction of the experience evaluation criteria could help to enhance the dialogue between the cultural heritage and the audience and promote the digital inheritance of the cultural heritage.

CHI's virtual art design takes information transmission as its original intention and purpose. It is a process of information deconstruction and reconstruction. It needs to cross the time stream of cultural heritage from the past to the present, and integrates the sensory experience to achieve cultural accumulation. Liz Sanders described three aspects of product demand: usability, accessibility, and satisfaction [6]. Usability makes achieve effective transmission of information based on accessibility (interaction) and satisfaction (aesthetic), and affects the user's subsequent emotions, memory, and learning. According to the three elements of product demand with combining cultural heritage virtual art display design, we give the basic requirements of UE for CHI virtual art. They are immersion that 
is the sense of reality in a given virtual environment, psychological feeling that is the emotion of the user in the operating process of the virtual environment and interactivity that is the information interaction between users and the virtual environment. Basing on the three main factors, we construct an architecture of evaluation criteria including detailed experience elements of the virtual art. As shown in Figure 3, the architecture adopts CHI ontology as the basis for information filtering and analysis, combines knowledge experience and presentation forms to optimize the knowledge transfer channel, and supports the visualization of CHI.

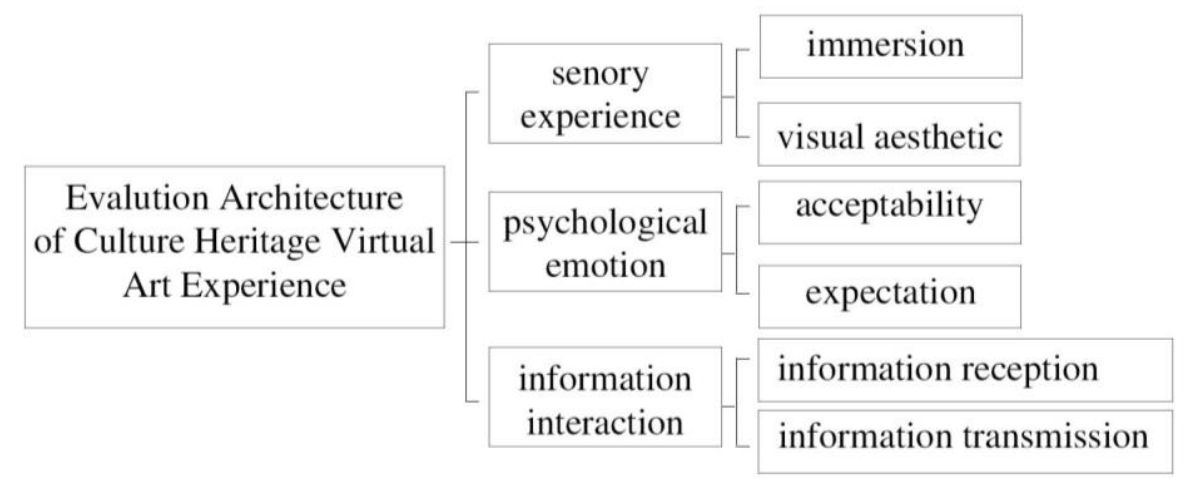

Fig. 3. Architecture of Evaluation Criteria on CHI Virtual Art Experience

Our understanding and practice about the evaluation criteria are as follow. Firstly, immersion is the sensory experience basis for the realization of all virtual reality art. People obtain information through the senses, and then pass this information to the brain. The brain connects the acquired information with the feelings when acquiring information through the psychological association, and finally the association influences the user's learning process. "Immersion" in the virtual art experience refers to the spirit's preoccupation [7]. It establishes a relatively closed hallucination space by inspecting the distance between the viewer and the display object. Image processing and visual arrangement are the key to immersive virtual reality. Therefore, we increase the sense of immersion in the simulation of the physical world, that is, a reasonable spatial layout that conforms to the laws of human perception enhances the aesthetic sense of the audience and creates a visual resonance of the overall environment. Secondly, psychological emotions drive and influence the concentration of the user's attention and goals. Antonio R. Damasio [8] defines feelings as "a perception of a specific physical state, together with the perception of a particular mode of thinking about a particular subject." Considering the acceptability of the experiencer, we designed a clear presentation of content and operational functions to meet the viewer's psychological expectation and balance the harmonious relationship between media devices and exhibits. Finally, the essence of virtual art is the transmission of information, and immersive interactivity is the main feature of virtual art that is different from other art forms and experiences. In order to avoid the audience from being unable to receive information accurately due to the complicated operations or other obstacles in the interaction, we guide the audience to in-depth operation through simple operations in the virtual environment. Through the combination of logical and cognitive design, we achieve a highly interactive procedure, which makes audiences receive cultural resources information from multiple dimensions through an interactive art experience with media devices.

\section{Qinling CHI virtual art experience evaluation}

The virtual restoration and display system of Qinling is an information interaction environment about cultural heritage of Qin dynasty. Based on real archaeology data, it aims to create an information-rich user experience in a virtual reality environment by comprehensively restoring the ground appearance of Emperor Qin's mausoleum. Experience, being personal and subjective, does not have a united evaluation criterion. Due to the differences in user's individual purposes and needs, it is difficult to measure virtual art experience with a single evaluation standard. Therefore, in order to assess whether the user is satisfied with the experience and guide the iterative design, the user's actual response and 
demand need to be obtained through the evaluation and analysis of the user testing results. Basing on the evaluation criteria, twelve users aging from 20 to 30 years old were chosen for the user experience test of virtual Qinling, and they all have experience at virtual reality system. Then the testing users completed questionnaires after the experience test. The statistical and analytical results of the evaluation test are shown in Table 1. In the table, the user's recognition of the relative importance degree of each experience element is donated $\mathrm{A}$ to $\mathrm{F}$ in order, in which $\mathrm{A}$ is the highest evaluation unit and $\mathrm{F}$ is the lowest evaluation unit.

Table 1. Results of the evaluation test of virtual Qinling user experience

\begin{tabular}{|c|c|c|c|c|c|c|}
\hline & \multicolumn{2}{|c|}{$\begin{array}{c}\text { Sensory experience } \\
\text { elements }\end{array}$} & \multicolumn{2}{|c|}{$\begin{array}{c}\text { Psychological emotion } \\
\text { elements }\end{array}$} & \multicolumn{2}{|c|}{$\begin{array}{c}\text { Information interaction } \\
\text { elements }\end{array}$} \\
\hline $\begin{array}{c}\text { Experimental } \\
\text { Users }\end{array}$ & $\begin{array}{c}\text { Visual } \\
\text { Aesthetic }\end{array}$ & Immersion & Acceptability & Expectation & $\begin{array}{c}\text { Information } \\
\text { transmitssio } \\
n\end{array}$ & $\begin{array}{c}\text { Information } \\
\text { acceptance }\end{array}$ \\
\hline User 1 & A & B & $\mathrm{F}$ & C & $\mathrm{E}$ & D \\
\hline User 2 & $\mathrm{~B}$ & $\mathrm{~A}$ & $\mathrm{D}$ & $\mathrm{E}$ & $\mathrm{F}$ & $\mathrm{C}$ \\
\hline User 3 & $\mathrm{C}$ & A & $\mathrm{E}$ & $\mathrm{B}$ & $\mathrm{F}$ & $\mathrm{D}$ \\
\hline User 4 & $\mathrm{D}$ & $\mathrm{B}$ & $\mathrm{C}$ & $\mathrm{F}$ & $\mathrm{E}$ & A \\
\hline User 5 & $\mathrm{C}$ & $\mathrm{F}$ & $\mathrm{A}$ & $\mathrm{D}$ & $\mathrm{B}$ & $\mathrm{E}$ \\
\hline User 6 & $\mathrm{~A}$ & $\mathrm{~B}$ & $E$ & $\mathrm{~F}$ & $\mathrm{D}$ & $\mathrm{C}$ \\
\hline User 7 & $\mathrm{~B}$ & $\mathrm{~A}$ & $\mathrm{D}$ & $\mathrm{E}$ & $\mathrm{C}$ & $\mathrm{F}$ \\
\hline User 8 & $\mathrm{~A}$ & $\mathrm{~B}$ & $\mathrm{~F}$ & $\mathrm{D}$ & $E$ & $\mathrm{C}$ \\
\hline User 9 & $\mathrm{D}$ & $\mathrm{B}$ & $\mathrm{A}$ & $\mathrm{F}$ & $\mathrm{E}$ & $\mathrm{C}$ \\
\hline User 10 & $\mathrm{~B}$ & A & $\mathrm{E}$ & $\mathrm{F}$ & $\mathrm{C}$ & $\mathrm{D}$ \\
\hline User 11 & $\mathrm{D}$ & $\mathrm{B}$ & $\mathrm{A}$ & $\mathrm{F}$ & $\mathrm{E}$ & $\mathrm{C}$ \\
\hline User 12 & $\mathrm{~B}$ & $\mathrm{~A}$ & $\mathrm{E}$ & $\mathrm{F}$ & $\mathrm{C}$ & $\mathrm{D}$ \\
\hline
\end{tabular}

From the Table 1, among the 12 users, there are 5 people who consider that the immersive feeling affects the user experience most, and 6 people think that immersion is relatively important. In addition, weight coefficient, which represents the importance of a certain criterion, could also reflect the impact degree of a certain criterion in the evaluation test. We set the value range of the weight coefficient is $(0,1)$ and the sum of all coefficient values is 1 . According to Table 1 , finally the value and distribution of each weight coefficient are obtained, as shown in Table 2.

Table 2. Weight coefficient distribution of each evaluation criterion

\begin{tabular}{|c|c|c|c|c|c|c|}
\hline & \multicolumn{2}{|c|}{$\begin{array}{c}\text { Sensory experience } \\
\text { elements }\end{array}$} & \multicolumn{2}{c|}{$\begin{array}{c}\text { Psychological emotion } \\
\text { elements }\end{array}$} & \multicolumn{2}{c|}{$\begin{array}{c}\text { Information interaction } \\
\text { elements }\end{array}$} \\
\cline { 2 - 6 } & $\begin{array}{c}\text { Visual } \\
\text { Aesthetic }\end{array}$ & Immersion & Acceptability & Expectation & $\begin{array}{c}\text { Information } \\
\text { transmitssio } \\
\mathrm{n}\end{array}$ & $\begin{array}{c}\text { Information } \\
\text { acceptance }\end{array}$ \\
\hline $\begin{array}{c}\text { Weight } \\
\text { coefficient } \\
\text { value }\end{array}$ & 0.15 & 0.28 & 0.12 & 0.10 & 0.13 & 0.22 \\
\hline
\end{tabular}

In the above test, we find that immersion bearing the biggest influence to the whole experience and information acceptance is the second important criterion. It shows that in the Qinling CHI virtual art experience, immersion and information acceptance are the dominant factors compared with the other four elements of criteria.

\section{Conclusion}

This paper discusses the VR display system of Emperor Qin's mausoleum and analyzes the CHI's virtual art experience and its evaluation criteria, provides a further design references for a better user experience of Qinling. From the perspective of user experience, a virtual Qinling space with rich cultural information will conduct viewers to experience the cultural charms of Qin dynasty, and a 
better immersive and interactive environment will turn the viewers' cultural experience into their own learning content and support the digital inheritance of Qingling cultural heritage.

\section{Acknowledgement}

This research was financially supported by China Postdoctoral Science Foundation (Grant NO. 2014M552479), Humanities and Social Science Youth Funded Project of MOE of China (Grant NO. 17YJCZH186), Natural Science Basic Research Plan in Shaanxi Province of China (Grant NO. 2013JQ8022), Scientific Research Project of Education Department of Shaanxi Province (Grant NO. 2013JK1181).

\section{References}

[1] J. Wu, Multiple and heterogeneous digital culture: presentation and display of Dunhuang Grottoes digital culture, Dunhuang Research, vol.1, pp.123-127, 2016.

[2] Y. T. Wang, Field reconstruction of Yuanmingyuan basing on augmented reality technology, Science and Technology Review, vol. 3, pp. 36-40, 2006.

[3] M. Z. Huang, Innovative practice on the exhibition of the National Palace Museum's cultural heritage: a summary of the design of the digital art museum of Duanmen, Identification and Appreciation of Cultural Relics, vol. 8, pp. 57-61, 2016.

[4] G. H. James, P. Joseph, The real economy: what consumers really want, CITIC Publishing House, pp. 15-19, 2010.

[5] J. J. Garrett, User experience elements, Mechanical Industry Press, pp. 42-45, 2011.

[6] D. A. Norman, Emotional design, Beijing Posts and Telecommunications Press, pp. 47-49, 2014.

[7] O. Grau, Virtual art, Tsinghua University Press, pp. 9-10, 2007.

[8] A. R. Damasio, Finding spinoza, Education Science Press, pp. 54, 2009. 